\title{
A Relevance Feedback Algorithm Combining Bayesian and FSRM
}

\author{
Zhang Shui-Li*, Dong Jun-Tang and Liu Li-Li \\ College of Physics and Electronic Information in Yan' an University, Yan' an City, Shaanxi Province, 716000, P.R. \\ China
}

\begin{abstract}
The semantic gap between low level visual features and high level semantic concepts, is an obstacle to the development of image retrieval. The semantic gap is narrowed by relevant feedback techniques to some extent. However, the image retrieval process with the relevant feedback technology also has many disadvantages such as too many feedback times or unsatisfactory feedback effect. In order to improve the relevance feedback method, a new relevance feedback strategy combining Bayesian and FSRM technology has been presented. The main approach was achieved firstly by assorting the image library with the Bayesian classifier compressing the image library; secondly, by searching the compressed image library with the FSRM; and lastly, by returning the worked out results. The experiment results illustrated the accuracy of the feedback method and showed it to be the best compared with FSRM algorithm and Bayesian algorithm.
\end{abstract}

Keywords: Bayesian(method), fuzzy semantic relevance matrix(FSRM), image retrieval, normal distribution, relevance feedback.

\section{INTRODUCTION}

With the development of multimedia technology and Internet, a vast multimedia information ocean appeared in front of people, which further lead to the super large image information base. The problem, how to effectively help people to find the needed information quickly and accurately, has become the core problem to be solved in the image retrieval system [1]. Relevance feedback (RF), which presented in text retrieval systems, has been introduced into the content based image retrieval (CBIR) field in the mid-1990s. RF makes the retrieval patterns from once to interactive on many occasions by the participation of the people into the information retrieval process, and has become an effective way to improve retrieval performance. In the interactive relevance feedback process, we only require the user to give the search results of the retrieval results of current system that are relevant, irrelevant, or the degree of correlation to the query image, and then the system will learn to give better retrieval results according to the user's feedback [2]. In recent years, many scholars have achieved some success in terms of relevance feedback. Vasconcelos and Lippmans [3, 4] used Gaussian mixture model on the DCT coefficients as feature representation, and used Bayesian inference for relevance feedback learning in the local features of the images. Their approach supports region query without the need for image segmentation. Aiming at the difficult problem of relevance feedback in less training samples, $\mathrm{Wu}$ et al proposed a relevance feedback probabilistic framework based on Bayesian rules. The method took into account all the samples (labeled and unlabeled samples) distribution characteristic when using labeled samples, so that retrieval performance was improved [4]. Guo Shihui proposed a relevance feedback

*Address correspondence to this author at the College of Physics and Electronic Information in Yan' an University, Yan' an City, Shaanxi Province, 716000, P.R. China; Tel: 18509110138, 18329918059; Fax: 0911-2332045; E-mail: zhangshuili74@163.com algorithm based on fuzzy semantic relevance matrix (FSRM). The algorithm adjusted the fuzzy semantic relevance matrix weights according to the user feedback on retrieval results, so as to capture the user's retrieval attempts, and then constantly modified the elements in the FSRM through learning the FSRM data, thus contacted the lowlevel visual features and high-level semantic features [5]. This paper presents a relevance feedback algorithm combined with the Bayesian classifier and fuzzy semantic relevance matrix. The algorithm firstly divides the image database into relevant and irrelevant image class categories by using Bayesian classifier, and then uses FSRM to retrieve. We can get the result closer to the user's needs by continuously updating the Bayesian classifier parameters and the weights of FSRM. The experimental results show that the proposed algorithm has a certain robustness.

\section{BAYESIAN DISTRIBUTION UNDER THE CONDI- TION OF MULTIVARIATE NORMAL DISTRIBU- TION [6]}

\subsection{Multivariate Normal Distribution}

Gauss distribution model, a general model of probability distribution, which is simple in operation, and the distribution of many events in the real world have great similarity with it. Assume that the vector $\mathrm{x}$ in $\mathrm{n}$ dimension space $\mathrm{Rn}$, meets the Gaussian distribution, the probability density function of $\mathrm{X}$ can be described as follows:

$$
P(x)=\frac{1}{(2 \pi)^{d / 2} \mid \Sigma^{1 / 2}} \exp \left(-\frac{1}{2}(x-u)^{T} \sum^{-1}(x-u)\right)
$$

Here, $x=\left(x_{1}, x_{2}, \ldots x_{d}\right)^{T}$ is a d-dimensional feature vector, $u=\left(u_{1}, u_{2}, \ldots u_{d}\right)^{T}$ is d-dimensional mean vector, $\Sigma$ is the $\mathrm{d} \times \mathrm{d}$-dimensional co-variance matrix, and $\Sigma^{-1}$ is the inverse matrix of $\Sigma,|\Sigma|$ is the determinant of $\Sigma$. 


\subsection{Bayesian Discrimination Principle of Multivariate Normal Distribution}

Bayesian classifier, based on Bayesian theorem, estimates the posterior probability through training a large number of samples, and classifies the sample into the class which has a large posterior probability, thereby the discriminant function of $\omega$ i class can be defined as follows:

$g_{i}(x)=P\left(x / \omega_{i}\right), \quad i=1,2, \ldots c$

Because Bayesian formula of total probability is the same for different classes, the discriminant function of various classes can also be defined as follows:

$$
g_{i}(x)=P\left(\omega_{i} / x\right) P\left(\omega_{i}\right), \quad i=1,2, \ldots c
$$

In the discriminant function, the prior probability $\mathrm{P}\left(\omega_{\mathrm{i}}\right)$ is constant and independent of feature vectors. Class conditional probability density $\mathrm{P}\left(\mathrm{x} / \omega_{\mathrm{i}}\right)$ meets certain probability distribution. Assuming that $\mathrm{P}\left(\mathrm{x} / \omega_{\mathrm{i}}\right)$ is in line with $\mathrm{d}$ dimensional normal distribution, then formula (3) can be expressed as follows:

$$
g_{i}(x)=\frac{P\left(\omega_{i}\right)}{(2 \pi)^{d / 2}\left|\Sigma_{i}\right|^{1 / 2}} \exp \left(-\frac{1}{2}\left(x-u_{i}\right)^{T} \sum_{i}^{-1}\left(x-u_{i}\right)\right)
$$

It is not convenient for the function to calculate because of its index. So, take the natural logarithm, get rid of the item independent of class, then formula (4) will be replaced by formula (5):

$g_{i}(x)=\ln P\left(\omega_{i}\right)-\frac{1}{2}\left(x-u_{i}\right)^{T} \sum_{i}^{-1}\left(x-u_{i}\right)-\frac{1}{2} \ln \left|\Sigma_{i}\right|$

Formula (5) shall be the final discriminant function. The data are classified by comparing discriminant function values.

\section{FSRM THEORY}

In 1965, the theory of fuzzy mathematics, was presented by Zadeh. It is used to study the problem of fuzzy uncertainty, which expanded the characteristics value scope of the set $\{0,1\}$ to the interval $[0,1]$ of continuous values. So, we can use value to the interval $[0,1]$ to represent a certain extent with which one object meets some concept. The closer the object corresponding value to 1 , the greater the degree of compliance with the concept; on the contrary, the smaller the degree of compliance. The Fuzzy Matrix A is defined as follows $[7,8]$ :

$$
\begin{gathered}
\mathrm{A}=\left(\mathrm{a}_{\mathrm{ij}}\right)_{\mathrm{m} \times \mathrm{n}}, \text { and } \mathrm{a}_{\mathrm{ij}} \in[0,1](\mathrm{i}=1,2, \ldots \mathrm{m} ; \mathrm{j}=1,2, \ldots, \mathrm{n}) \\
R(i, j) \in[0,1], R(i, j)=1, \operatorname{and} R(i, j)=R(j, i), i, j=0,1, \ldots, N-1
\end{gathered}
$$

Here, $\mathrm{N}$ is the number of images in the image library. $R(i, j)$, the direct similarity, reflects the similarity between two images $i$ and $j$. FRSM is a Fuzzy similar matrix according to the definition of fuzzy matrix.

The size of FSRM is decided by the number of images in the image library according to the image retrieval algorithm based on FSRM mentioned in [8]. Thus, the FSRM is a very large matrix, whose corresponding computation will be enormous. In order to reduce the size of the FSRM, this pa- per firstly classifies image library into two classes of relevant and irrelevant, and then a FSRM is established for each class, thus the size of FSRM is greatly reduced.

\section{FEEDBACK ALGORITHM BASED ON COMBINA- TION OF BAYESIAN AND FSRM}

\subsection{Bayesian Classification Under Multivariate Normal Conditions}

According to the formula (5), for the discriminant function of Bayesian classification under multivariate normal conditions, there are three parameters involved in the discriminant function of class $i$ : that is the mean ui, covariance matrix $\Sigma \mathrm{i}$ and the prior probability $\mathrm{P}\left(\omega_{\mathrm{i}}\right)$. In the relevance feedback process, assuming the image library is labeled as two classes of relevant and irrelevant images, each Bayesian classifier parameter is updated according to the user feedback, and then we can continue to improve the performance of the two-class classifier with the updated parameters. Formula (7) is the three parameter update equations for two Bayesian classifier:

$$
\begin{aligned}
P_{r} & =\frac{N_{r}}{N_{r}+N_{n}}, & P_{n} & =\frac{N_{n}}{N_{r}+N_{n}} \\
u_{r} & =\operatorname{mean}\left(I^{+}\right), & u_{n} & =\operatorname{mean}\left(I^{-}\right) \\
\Sigma_{r} & =\operatorname{cov}\left(I^{+}\right), & \Sigma_{n} & =\operatorname{cov}\left(I^{-}\right)
\end{aligned}
$$

Here, $P_{r}$ and $P_{n}$ represent respectively the prior probability of the relevant and irrelevant image classes; $\mathrm{N}_{\mathrm{r}}$ and $\mathrm{N}_{\mathrm{n}}$ are respectively the number of relevant and irrelevant images; $\mathrm{U}_{\mathrm{r}}$ and Un are the mean of two classes; I+ and I- respectively represent the relevant and irrelevant images; $\Sigma \mathrm{r}$ and $\Sigma_{\mathrm{n}}$ are respectively the two classes of co-variance matrix.

It is difficult to get enough feedback information in the actual retrieval, partly because the user does not want to interact too many times in the search process, and also because the number of images is not comparable to the image feature dimension in the same image library, which makes the covariance matrix difficult to be estimated accurately, so the co-variance matrix is reduced to a diagonal matrix as follows:

$$
\operatorname{diag}\left\{\delta_{k}^{2}\right\}, \text { here, } \delta_{k}(m)=\sum_{k}(m, m) .
$$

After the search process using the formula (7), the parameters of the two classifiers are updated respectively. The user can label the image library into two classes of relevant image database and irrelevant image database according to the correlation between the query image and database images.

According to the Bayesian classifier update equations, we can get the experimental procedures using the Bayesian classifier for retrieval; the specific steps are:

(1) According to user needs, choose the former K images as the search results returned to the user. The users label the $\mathrm{k}$ images as the current relevant image database $\mathrm{I}_{1}^{+}$, the remainder being irrelevant image database $\mathrm{I}_{1}^{-}$.

(2) The parameters of the relevant image class and irrelevant image class are updated respectively after using formula 
(7). Then, the new classification results can be produced: relevant image class: $\mathrm{I}^{+}=\left(\mathrm{I}^{+} \cup \mathrm{I}^{+}{ }_{1}\right)-\mathrm{I}^{-}$, irrelevant image class: $\mathrm{I}^{-}=\left(\mathrm{I}^{-} \cup \mathrm{I}_{1}^{-}\right)-\mathrm{I}^{+}$.

(3) Each of the relevant and irrelevant image class will have its own classification discriminant functions $\mathrm{g}_{\mathrm{r}}(\mathrm{x})$ and $\mathrm{g}_{\mathrm{n}}(\mathrm{x})$ after each cycle of updating parameters. With the image feature parameters of image library for the two discriminant functions, if $g_{r}(x)>g_{n}(x)$, put $x$ attributable to the relevant image library. Thus, after Bayes discriminant, the $\mathrm{U}+$, current relevant image library of this feedback, will be obtained.

(4) In relevant library of images, each image has corresponding score: $\operatorname{score}\left(\mathrm{I}_{\mathrm{i}}\right)=\mathrm{f}\left(\mathrm{x}_{\mathrm{i}}\right)$, the larger the score, the closer the image similarity to the query image. Sort the images in the relevant image library by the score, and the first K maximum score images will feedback to the user.

(5) If the user is satisfied, then save the results, and terminate the retrieval. Otherwise, the user submits the labeled image, go to the second step again until the feedback results meet the user's need.

\subsection{FSRM Feedback Algorithm}

For each class of image library, $R(i, j)$, the similarity of images $i$ and $j$, is represented by the element of FSRM, which is initialized by a symmetric matrix :

$0 \leq R(i, j) \leq 1(i, j=1,2, \ldots, N)$ if $i=j$ then: $R(i, j)=1$; otherwise : $R(i, j)=R(j, i)(i, j=1,2, \ldots, N)$

Here, $\mathrm{N}$ is the number of each image library. Because there are some similarities between the images for each image category, the initial value of FSRM will be set to be the vaguest value of 0.5 between $0-1$, that is $\mathrm{R}(\mathrm{i}, \mathrm{j})=0.5(\mathrm{i} \neq \mathrm{j}$ and $\mathrm{i}, \mathrm{j}=1,2, \ldots, \mathrm{N})$.

During FSRM feedback process, for the relevant image class $\left(\mathrm{I}^{+}\right)$in the same image library, the corresponding weights in FSRM are adjusted according to the formula (9), or do not modify; for irrelevant image class $\left(\mathrm{I}^{-}\right)$in the same image library, the corresponding weights in FSRM are adjusted according to the formula (10).

$$
\begin{aligned}
& R(i, j)_{\text {new }}=R(i, j)_{\text {old }}+\alpha\left(1-R(i, j)_{\text {old }}\right) \\
& R(i, j)_{\text {new }}=R(i, j)_{\text {old }}-\beta\left(1-R(i, j)_{\text {old }}\right)
\end{aligned}
$$

Here, $\alpha$ and $\beta$ respectively express the degree of parame- ter weights increased or decreased, and satisfy $\alpha+\beta=1$. Here, select $\alpha=0.35, \beta=0.65$.

With FSRM weight adjustment formulas, we can get the experimental procedures using FSRM for retrieval, the specific steps are:

(1) Initialize the FSRM.

(2) After research based on low-level visual features, choose the former $\mathrm{K}$ images as the search results returned to the user. The users label the $\mathrm{k}$ images as the current relevant image database $\mathrm{I}_{1}^{+}$, the remainder being irrelevant image database $\mathrm{I}_{1}^{-}$.

(3) According to the formulas (9) and (10), adjust respectively each class of the image library weights corresponding value in FSRM.

(4) Partial weights of each image library in FSRM have been made from an initial value of 0.5 to a more realistic semantic value after training through a limited times of step 3. That is, a certain degree of similarity has been established between images in each image class, and then return the results back to the user.

(5) According to the user feedback, mark the corresponding image in FSRM weight from big to small order, $\alpha$ and return the most similar images to the user. If the user is satisfied, then save the results, $\alpha$ and terminate the retrieval. Otherwise, go to the second step again until the feedback results meet the user's needs.

\subsection{Feedback Technology of Bayesian and FSRM Com- bination}

By combining Bayesian and FSRM, this paper proposed a feedback algorithm. The specific steps included are: Firstly, obtain relevant and irrelevant image class by combining the labeling results of current and former feedback. Secondly, compress the images in the image database by using the Bayesian classifier under normal distribution, and establish relevant image class and irrelevant image class. Finally, compute the semantic relatedness between images by using FSRM algorithm for each image class respectively, and return the results to the user. If the user is satisfied, then save the results, and terminate the retrieval. Otherwise, continuously update the parameters of Bayesian classifier and FSRM according to the formulas (7), (9) and (10) , and retrieve again until the feedback results shall meet the user's need, and then return the final result. The process is shown

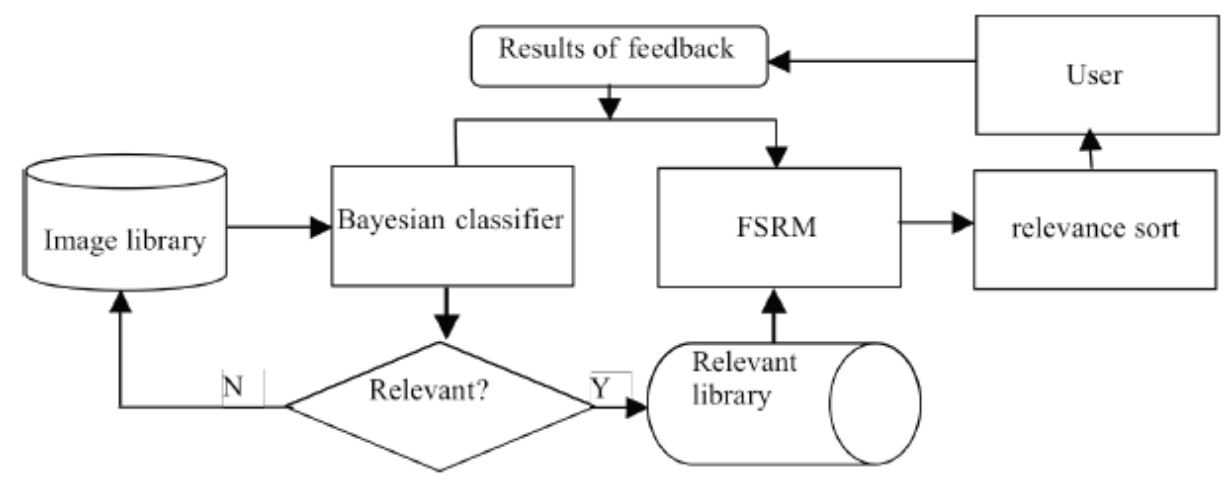

Fig. (1). Framework of image retrieval system based on combining Bayesian and FSRM. 


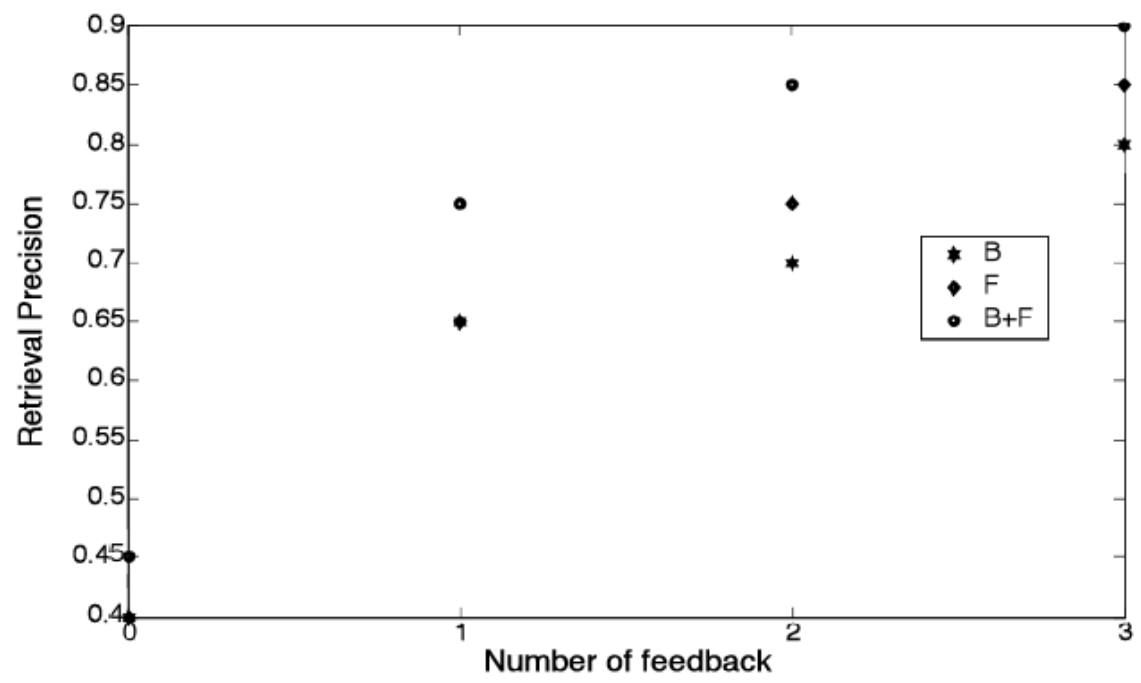

Fig. (2). Feedback accuracy of Top 20.



Fig. (3). Feedback accuracy of Top 25.

in Fig. (1), the specific algorithm process is as follows:

(1) Initialize the FSRM.

(2) According to the user's needs, choose the former K images as the search results returned to the user. The users label the $\mathrm{k}$ images as the current relevant image database $\mathrm{I}+1$, the remainder being irrelevant image database I - 1 .

(3) The parameters of the relevant image class and irrelevant image class are updated respectively after using formula (7). Then, the new classification results can be produced: relevant image class: $\mathrm{I}^{+}=\left(\mathrm{I}^{+} \cup \mathrm{I}^{+}{ }_{1}\right)-\mathrm{I}^{-}$, irrelevant image class: $\mathrm{I}^{-}=\left(\mathrm{I}^{-} \cup \mathrm{I}_{1}^{-}\right)-\mathrm{I}^{+}$.

(4) For the relevant image class $\left(\mathrm{I}^{+}\right)$in the same image library, the corresponding weights in FSRM are adjusted according to the formula (9), or do not modify. For irrelevant image class $\left(\mathrm{I}^{-}\right)$in the same image library, the corresponding weights in FSRM are adjusted according to the formula (10).

(5) Partial weights of each image library in FSRM have been made from an initial value of 0.5 to a more realistic semantic value after training through a limited times of step 3-4. That is, a certain degree of similarity has been established between images in each image class, and then return the results back to the user.

(6) Mark the corresponding image in FSRM weight from big to small order, and return the most similar images to the user. If the user is satisfied, then save the results, and terminate the retrieval. Otherwise, go to the second step again until the feedback results to meet user needs.

\section{EXPERIMENTAL RESULTS AND ANALYSIS}

In experiments, we selected three semantic class image libraries related to figures, flowers and landscapes respectively, in which each class included 50 images. Then, the primary color feature of each of HSV color space was extracted by computing the HSV color histogram, namely the HSV mean. Thus a $1 \times 3$-dimensional color characteristics could be obtained. 
During the retrieval process, the user can retrieve each semantic class image library. Therefore, select 3 as the feedback times by taking into account the user's patience. Use the common evaluation criteria (average precision) for filing image retrieval to evaluate the performance of the system. In the experiments for each type of image library, we counted the number of relevant images ranked in the top 20 and top 25 for each feedback result, and calculated the feedback accuracy respectively. Finally, the average feedback accuracy was calculated for 3 feedback times. Feedback accuracy is defined as formula (11):

$P=a / b$

Here, a is the number of relevant images ranked in the top $\mathrm{K}, \mathrm{b}$ is the number of irrelevant images ranked in the top $\mathrm{K}$.

Figs. (2) and (3) show the average recall curves through 3 times of feedback by respectively using three ways for retrieval: B (Bayesian), FR (fuzzy to relevance matrix), and B $+\mathrm{F}$ (combining B and F). The curves show that the algorithm combining Bayesian and FSRM is the best among the three, and further demonstrate the effectiveness of the algorithm proposed in this paper.

\section{CONCLUSION}

Relevance feedback plays an important role in the image retrieval. The key problem to solve is how to improve feedback accuracy at reducing feedback times. A relevance feedback algorithm combining Bayesian and FSRM has been proposed in this paper. Firstly, the algorithm classifies image library into two classes of relevant and irrelevant by multidimensional Bayesian classifier under normal conditions. Then, the two compressed image libraries are searched with the FSRM. It has been observed through the experiment re- sult that the proposed algorithm improved the retrieval accuracy at reducing the feedback times, and narrowed the semantic gap between low level visual features and high level semantic concepts to some extent.

\section{CONFLICT OF INTEREST}

The authors confirm that this article content has no conflict of interest.

\section{ACKNOWLEDGEMENTS}

This work was financially supported by the Shaanxi Natural Science Foundation (2014JM8357) and Yan'an University Youth Foundation (YDQ2014-37).

\section{REFERENCES}

[1] X.Y Tan, Z.X. Sun, and F.Y. Zhang, "Relevance feedback in content-based image retrieval: the state of the art", Journal of Nanjing University (Natural Sciences), vol. 40, pp. 639-648, 2004.

[2] S.L. Sun, and X.Y. Lin, "Relevance feedback algorithm based on memory support vector machines", Computer Science, vol. 38, pp. 256-258, 2011

[3] N. Vasconcelos, and A. Lippman, " Learning from user feedback in image retrieval systems", Computer Vision - ECCV 2000,Lecture Notes in Computer Science, vol. 1842, pp. 33-47, 2000.

[4] H.X. Bai, Relevance Feedback Technology Based on CBIR. Hebei University of Science and Technology: China, 2008.

[5] S.H. Guo, and M. Yang, "Relevance feedback algorithm based on fuzzy semantic relevance matrix in image retrieval", Computer Science, vol. 39, pp. 540-542, 2012.

[6] C.J Chen, and J. Hou, "A relevance feedback strategy combining bayesian and SVM", Journal of Chengdu University of Information Technology, vol. 27, pp. 32-37, 2012.

[7] Z.Z. Peng, and Y.Y. Sun, Fuzzy Mathematics and its Application, Wuhan University Press: Wuhan, pp. 75-80, 2003.

[8] H.J. Jin, Research on Image Retrieval Based on Semantic Feature, Harbin Institute of Technology: China, 2006.

\footnotetext{
Received: September 16, 2014

(C) Shui-Li et al.; Licensee Bentham Open.
}

Revised: December 23, 2014

Accepted: December 31, 2014

This is an open access article licensed under the terms of the Creative Commons Attribution Non-Commercial License (http://creativecommons.org/licenses/by-nc/4.0/) which permits unrestricted, non-commercial use, distribution and reproduction in any medium, provided the work is properly cited. 